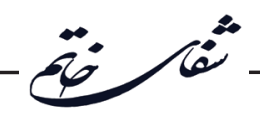

\title{
Comparison of Frequency and Intensity of Stressors in Infertile Couples Undergoing Intrauterine Insemination Treatment, Intra-Cytoplasmic Sperm Injection Treatment and without Treatment
}

\author{
Fereshteh Dadfar ${ }^{1}$, Mahboubeh Dadfar ${ }^{2 *}$, Pir Hossien Kolivand ${ }^{3}$ \\ ${ }^{1}$ Department of Biology, Payam Noor University, Iran \\ ${ }^{2}$ Department Clinical Psychology, School of Behavioral Sciences and Mental Health, International Campus, Iran University of Medical \\ Sciences, Tehran, Iran \\ ${ }^{3}$ Shefa Neuroscience Research Center, Khatam Alanbia Hospital, Tehran, Iran
}

\section{ABSTRACT}

Introduction: Infertility is a personal crisis. There are different assisted reproductive techniques in the treatment of infertility. Anxiety and stress have been shown to be markedly higher in infertile couples. This study was aimed to compare the frequency and intensity of stressors in infertile couples undergoing intrauterine insemination (IUI) treatment, Intra-cytoplasmic sperm injection (ICSI) treatment and without treatment (control group). Materials and Methods: Thirty seven infertile couples undergoing IUI, ICSI and without treatment were selected by non-random convenient sampling from the cases admitted to Rooyan Infertility Clinic. They completed demographic information sheet, and the Stressors Scale. Results: The results showed that there was no significant correlation between duration of infertility with frequency and intensity of stressors. There was no significant correlation between frequency and intensity of stressors with infertility factors. There was also no significant difference in the frequency and intensity of stressors between men and women. There were no differences in the frequency and intensity of stressors between ICSI and IUI with control group. Data showed that there was a significant difference in the frequency of stressors with the group of IUI treatment as well as in the frequency of stressors between ICSI and IUI with control group. Findings demonstrated that there was significant difference between IUI with and without ICSI groups in the frequency and intensity of stress. Conclusion: IUI, ICSI and control groups as the assisted reproductive techniques can be stressful. Long-term planning of mental health services for infertile couples, identifying couples at risk, convincing couples to refer for psychological assessment, psychotherapy by clinical psychologists, and drug therapy by psychiatrists are suggested.

\section{Key words:}

1. Infertility

2. Reproductive Techniques

3. Sperm Injections, Intracytoplasmic

*Corresponding Author: Mahboubeh Dadfar

E-mail:mahboubehdadfar@yahoo.com 
مقايسُٔ فراوانى و شدت عوامل استرسزا در زوجهاى نابارور تحت درمان تلقيح داخل رحمى، تحت درمان تلقيح درون سيتو يلاسمى اسيرم و بدون درمان

\author{
فرشته دادفر '، محبوبه دادفر זّ، ييرحسين كوليوند” \\ أروه زيستشناسى، دانشعاه يِيام نور، ايران \\ rكروه روانشناسى بالينى، دانشكده علوم رفتارى و سلامت روان، يرديس بين الملل، دانشعاه علوم يزشكى ايران، تهران، ايران \\ "مركز تحقيقات علوم اعصاب شفا، بيمارستان خاتمالانبياء، تهران، ايران
}

كليد وازهها:

1

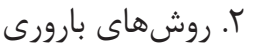

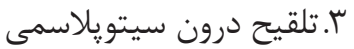

|
مقدمه: نابارورى يك بحران شخصى است. روشهاى مختلف كمك بارورى در درمان نابارورى وجود دارد دارد.

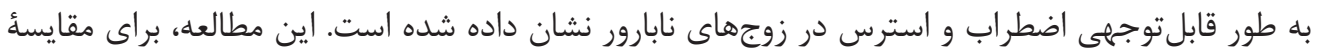

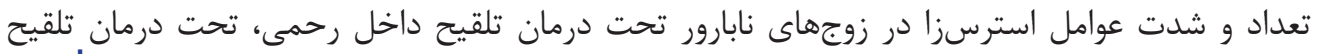

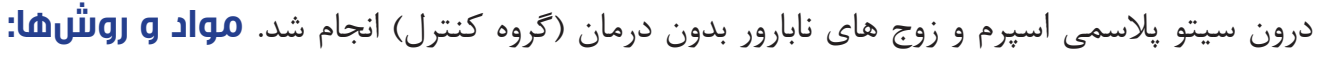

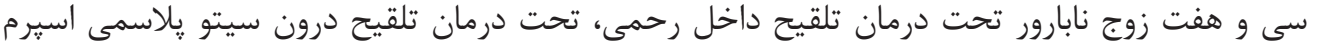

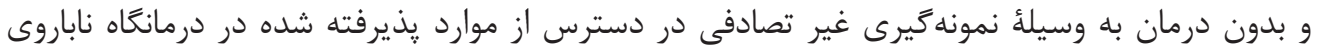

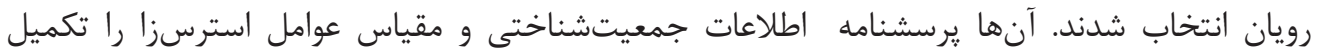

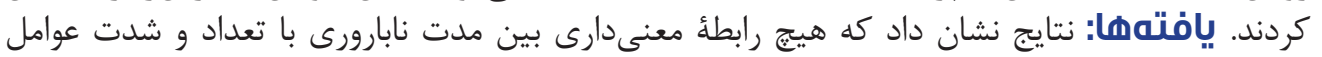

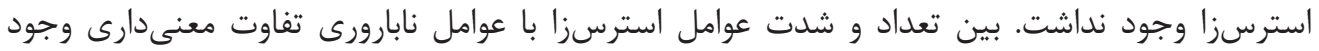

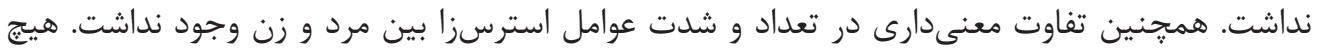

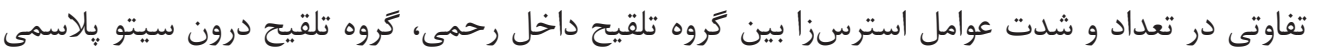

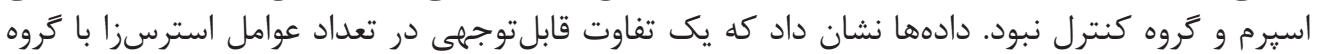

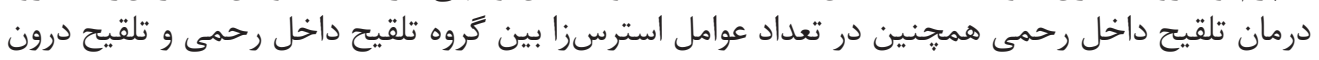

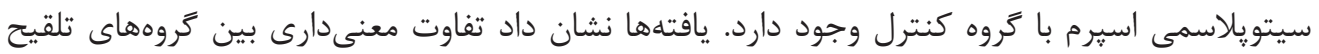

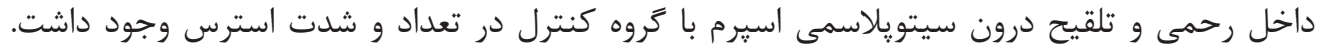

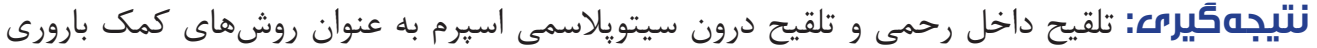

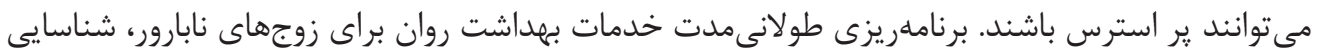

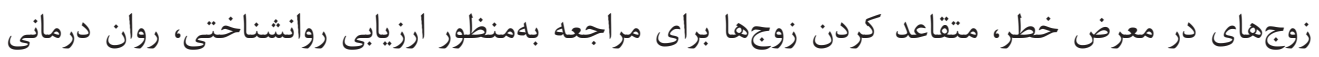

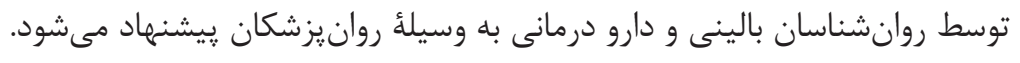


در سـالهاى اخيــر، ابعـاد روانشـــاختى نابـارورى در ميــان

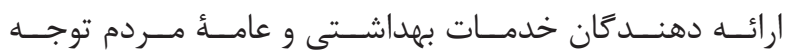

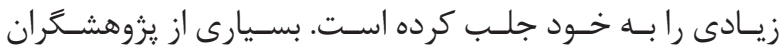

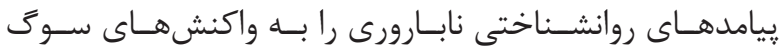

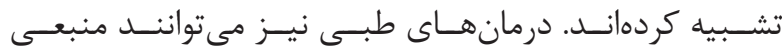

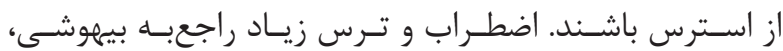

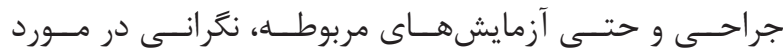

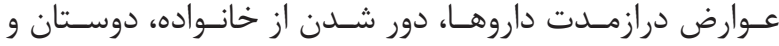

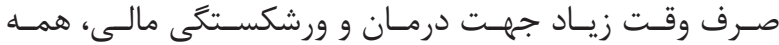

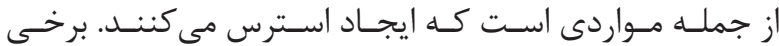

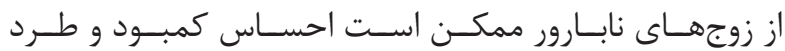

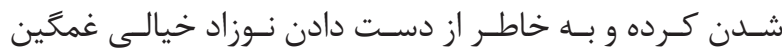

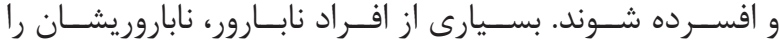

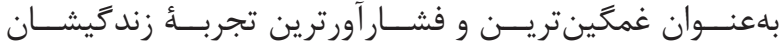

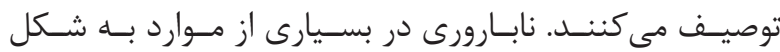

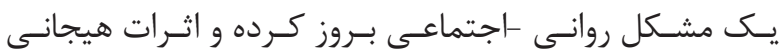

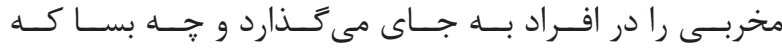

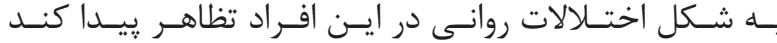

(1, $\mathrm{V}-1 \cdot)$

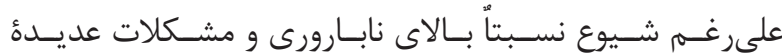

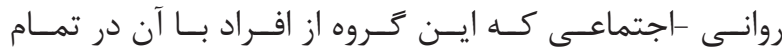

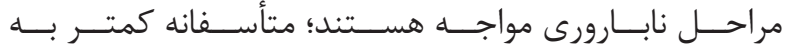

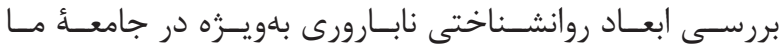

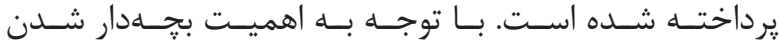

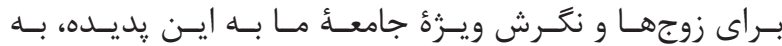

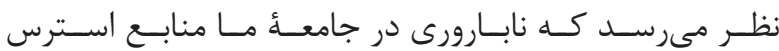

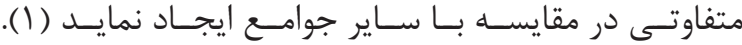

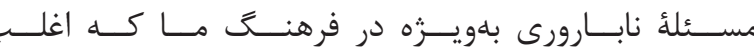

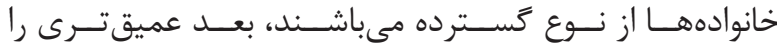

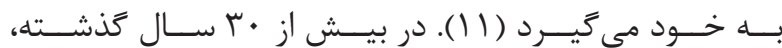

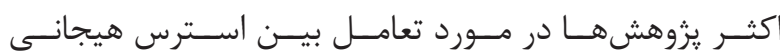

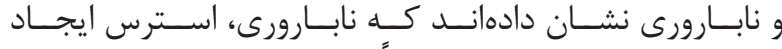

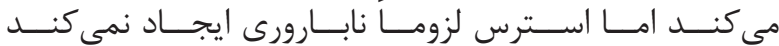

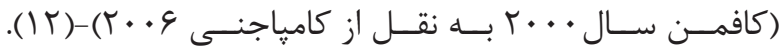

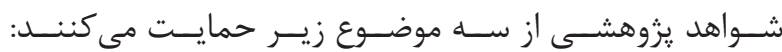

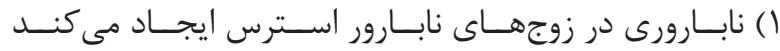

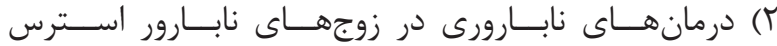

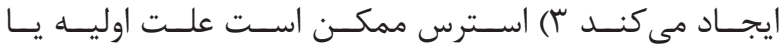

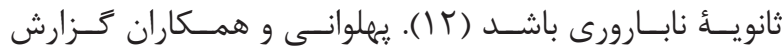

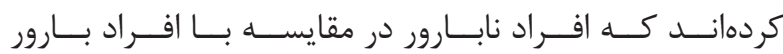

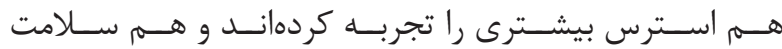

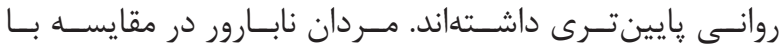

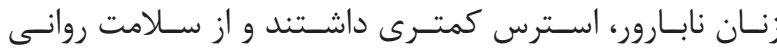

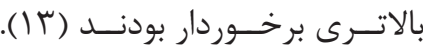

${ }^{1}$ In vitro fertilization

${ }^{2}$ Intracytoplasmic sperm injection

${ }^{3}$ Intrauterine insemination

${ }^{4}$ Ovum donation

${ }^{5}$ Surrogacy

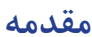

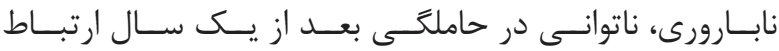

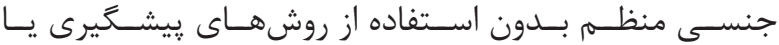

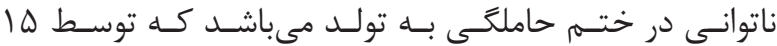

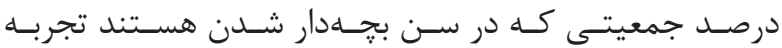

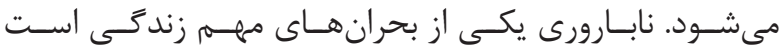

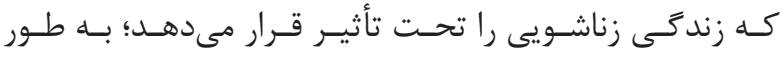

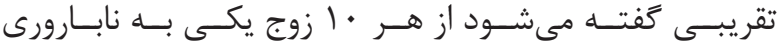

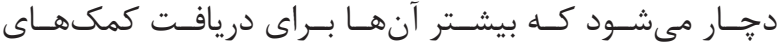

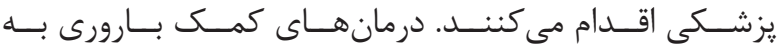

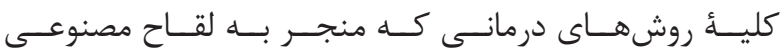

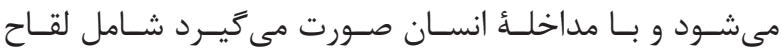

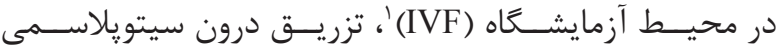

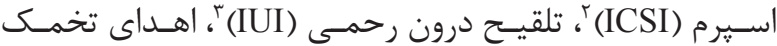

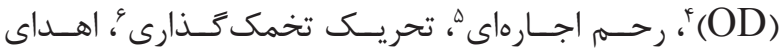

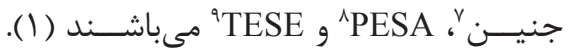
در كنفرانسس جهانسى جمعيست و توسـعه كسه در سـال 1994

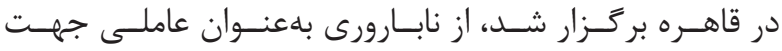

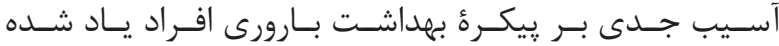

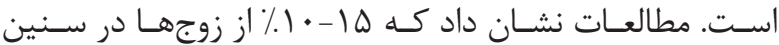

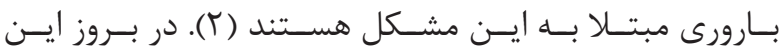

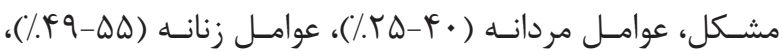

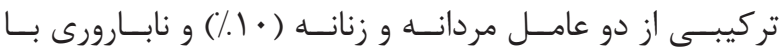

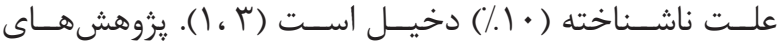

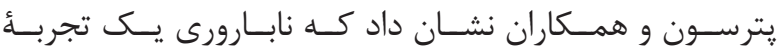

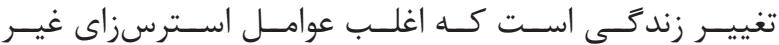

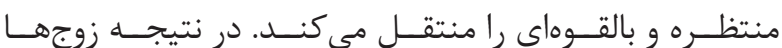

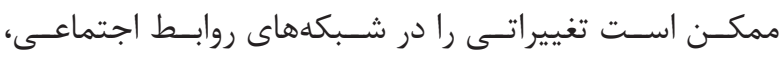

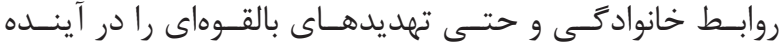

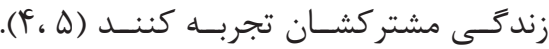

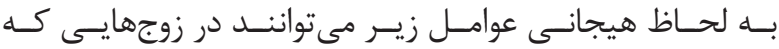

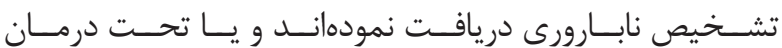

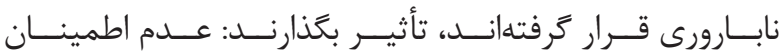

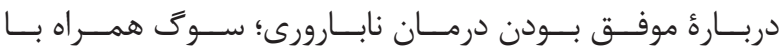

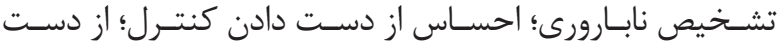

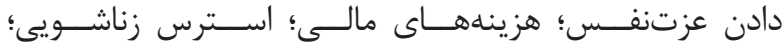

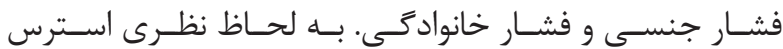

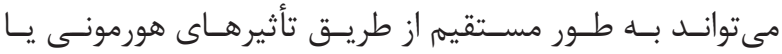

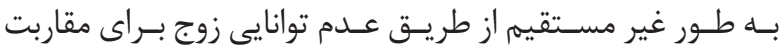

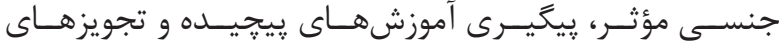

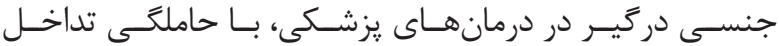

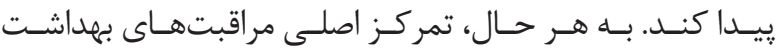

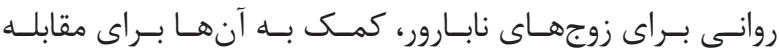

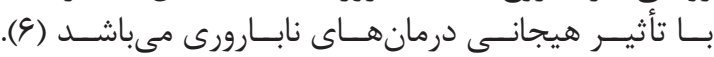

${ }^{6}$ Induction ovulation

${ }^{7}$ Embryo donation

${ }^{8}$ Percutaneous epididymal sperm aspiration

${ }^{9}$ Testicle epididymal sperm extraction 


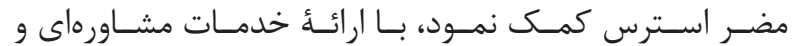

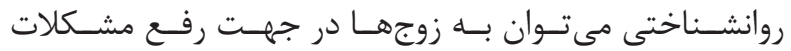

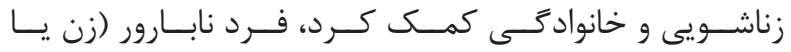

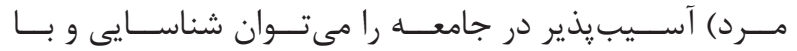

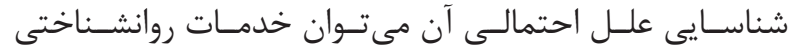

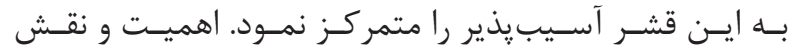

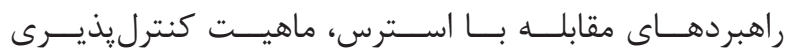

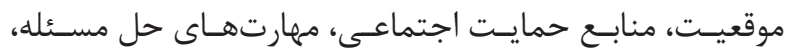

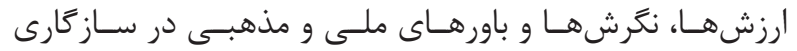

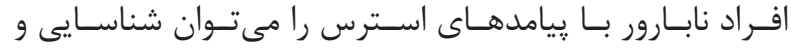

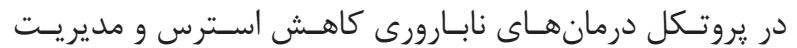

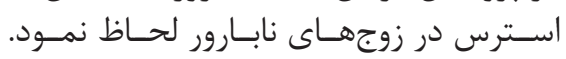

مواد و روشها

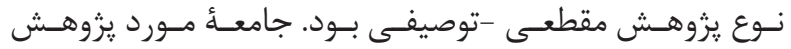

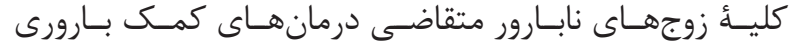

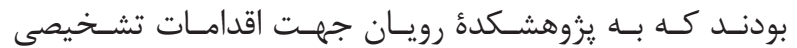

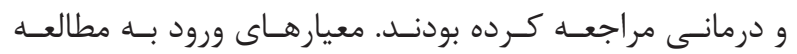

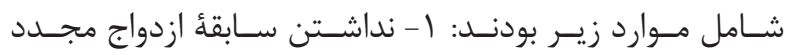

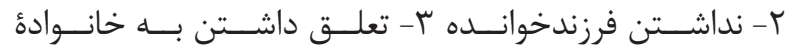

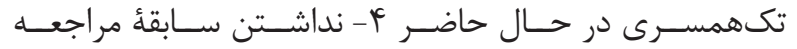

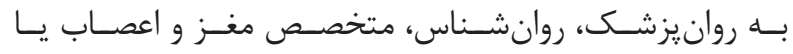

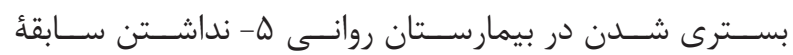

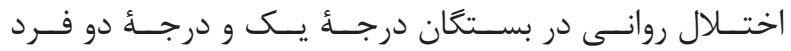

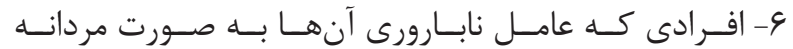

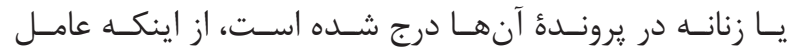

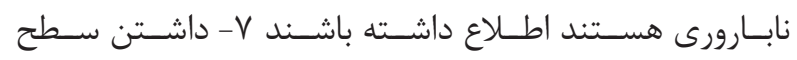

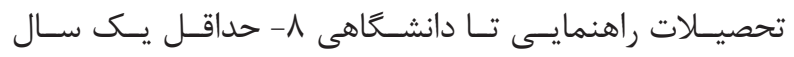

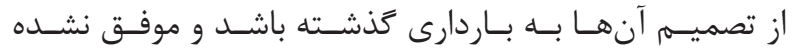

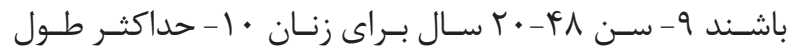
مـدت ازدواج ها ســال.

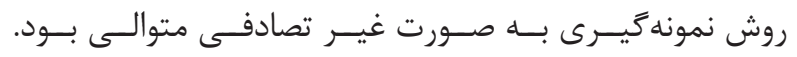

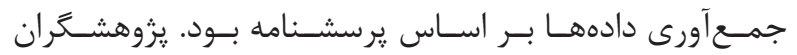

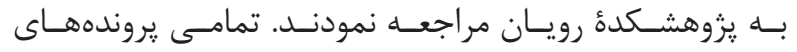

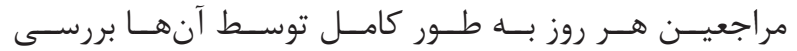

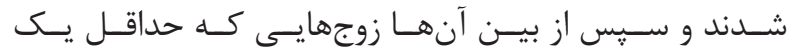

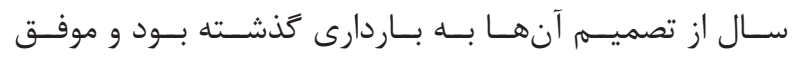

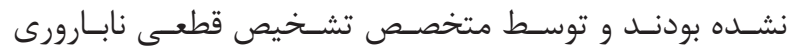

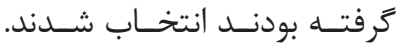

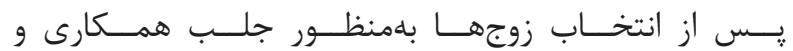

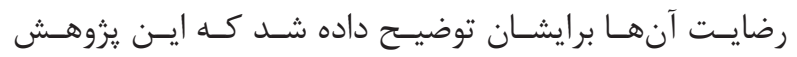

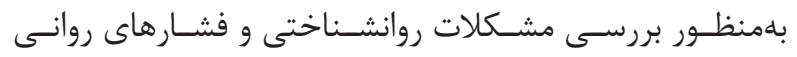

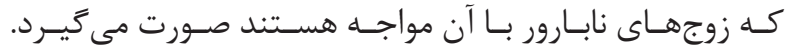

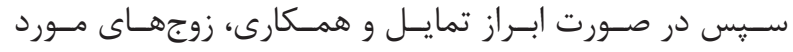

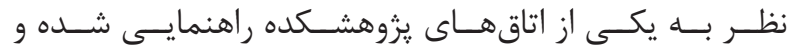

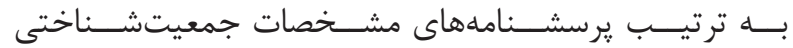

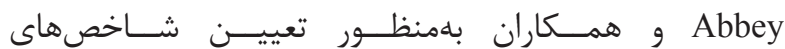

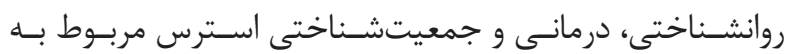

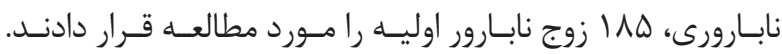

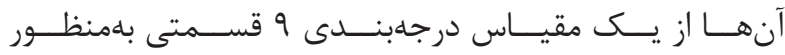

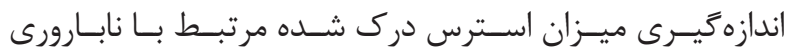

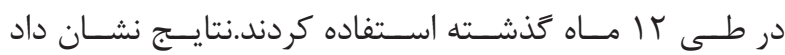

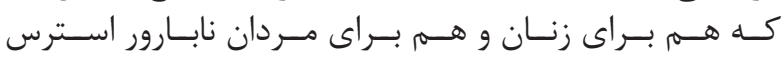

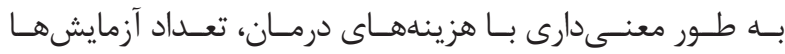

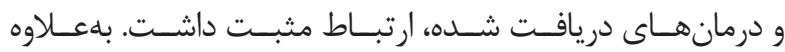

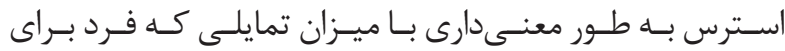

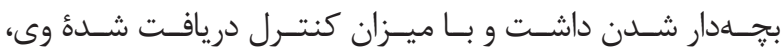

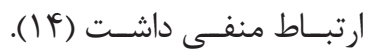

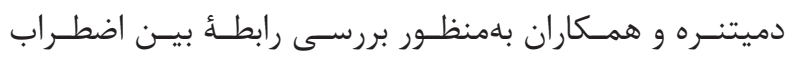

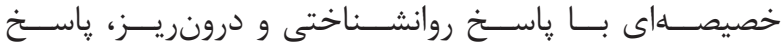

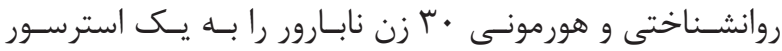

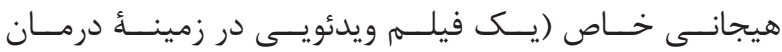

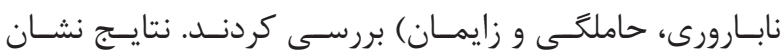

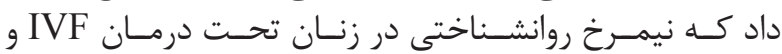

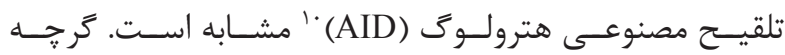

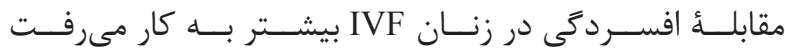

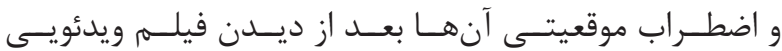

بيشـتر بـود (ه) (1).

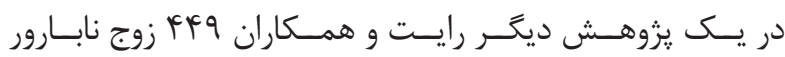

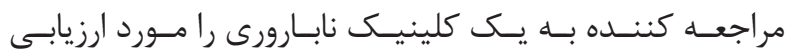

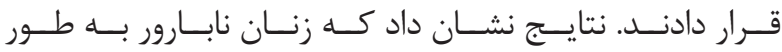

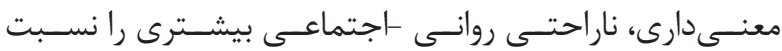

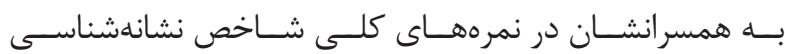

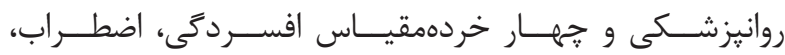

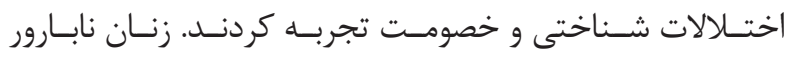

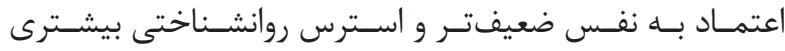

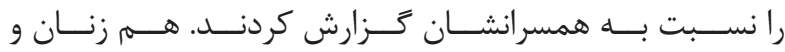

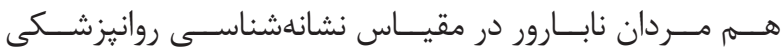

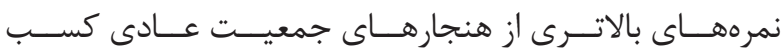

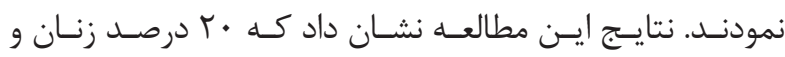

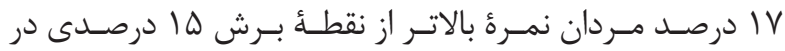

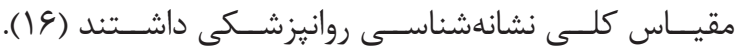

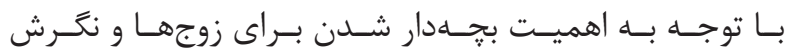

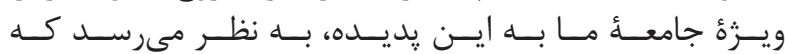

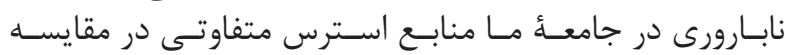

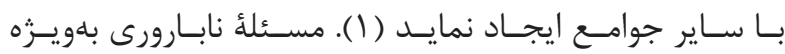

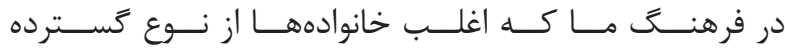

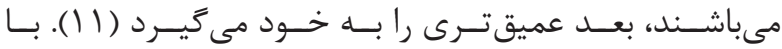

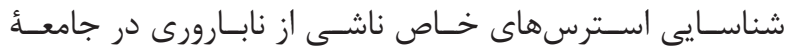

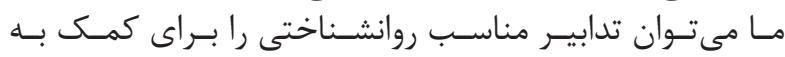

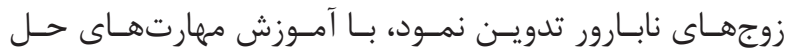

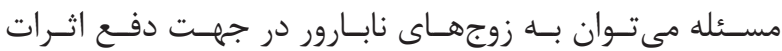

${ }^{10}$ Artificial insemination by donor 
تجزيه و تحليل دادهها

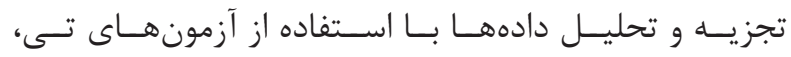

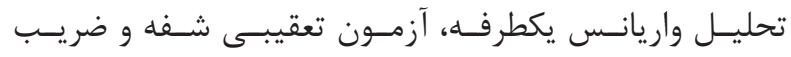

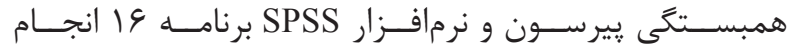

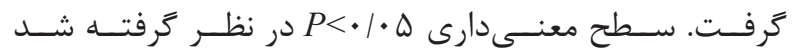

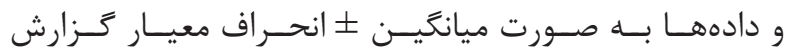

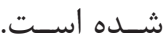

بافته ها

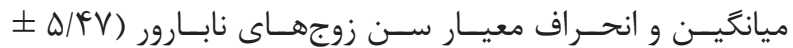

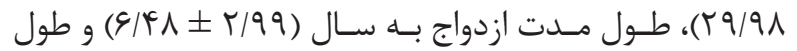

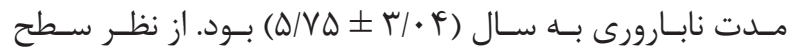

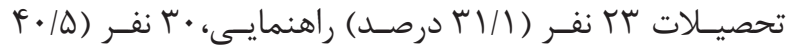

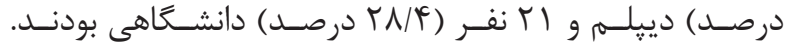

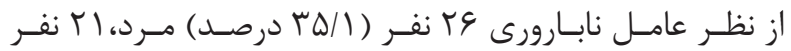

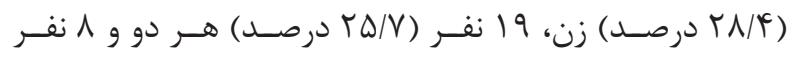

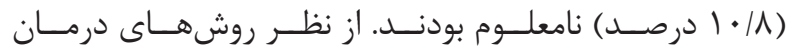

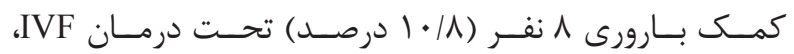

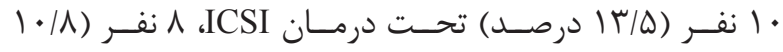

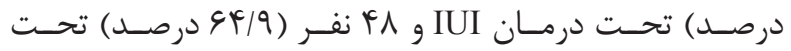

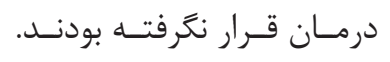

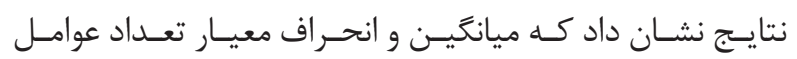

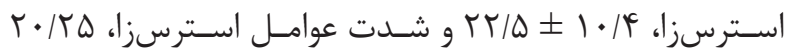

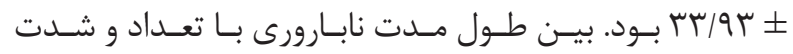

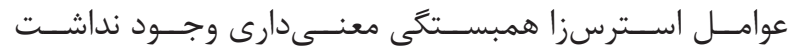

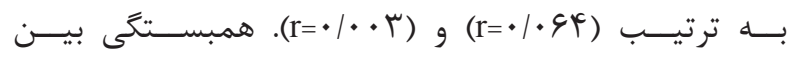

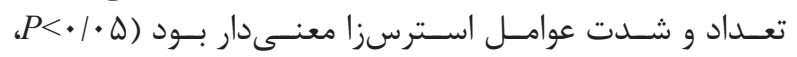

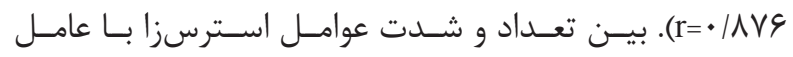

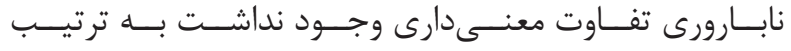

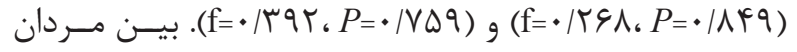

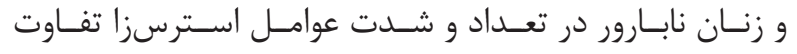

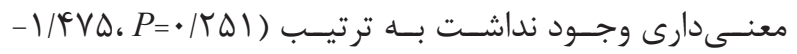

$$
\text { . }(\mathrm{f}=-\cdot / 9 \Lambda 1 ، P=\cdot / 1 \mathrm{r} \vee)_{g}(\mathrm{f}=
$$

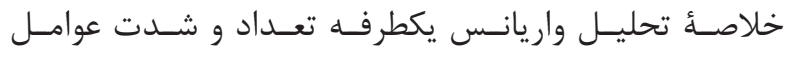

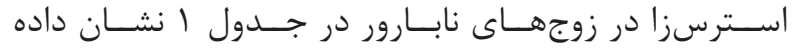

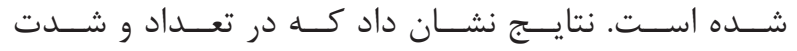

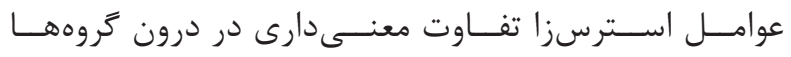

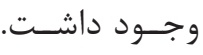

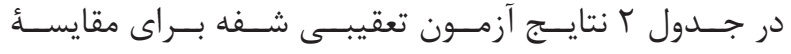

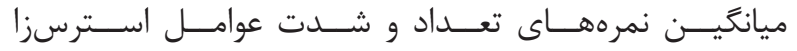

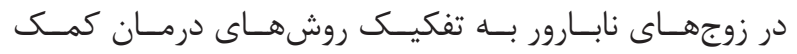

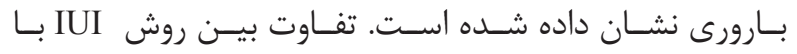

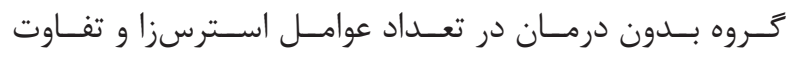

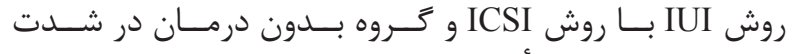

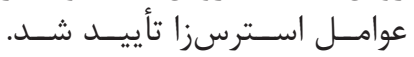

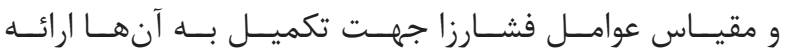

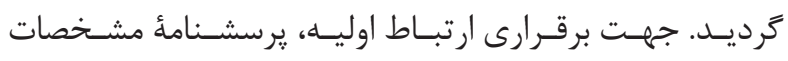

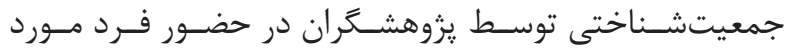

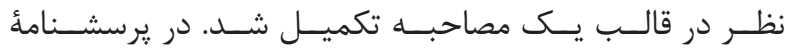

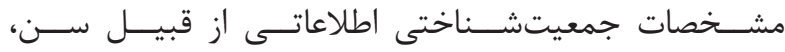

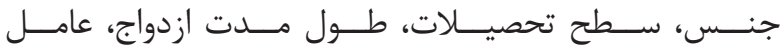

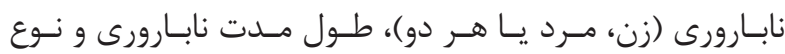

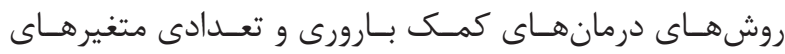

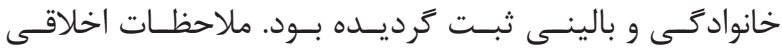

$$
\text { شـامل مـوارد زيــر بــود. }
$$

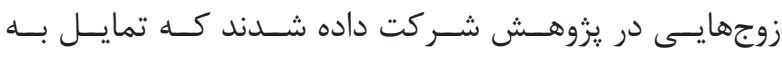

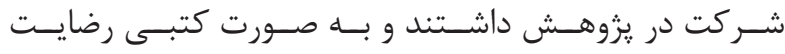

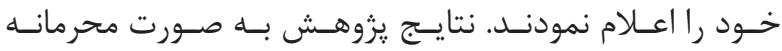

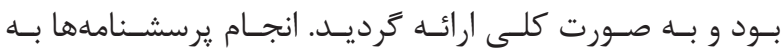

$$
\text { صـورت رايـعان بـود. }
$$

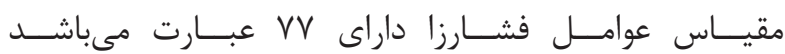

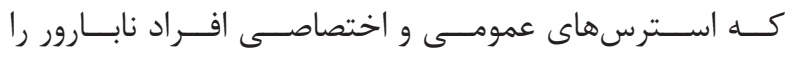

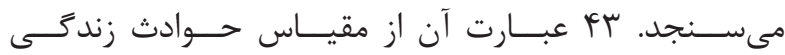

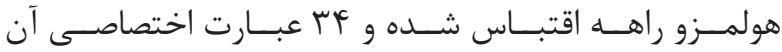

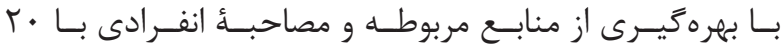

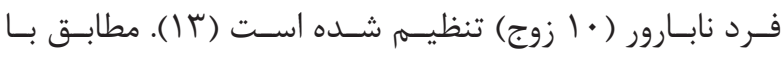

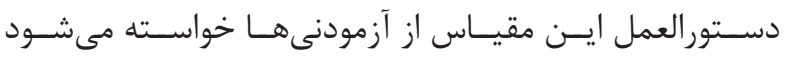

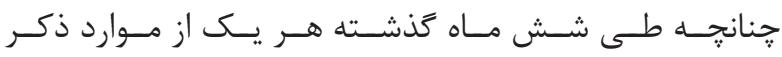

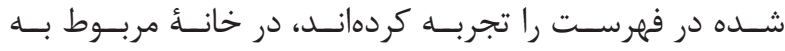

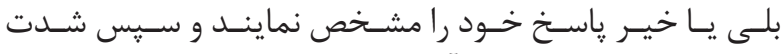

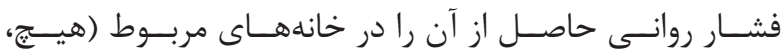

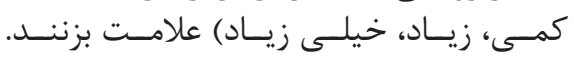

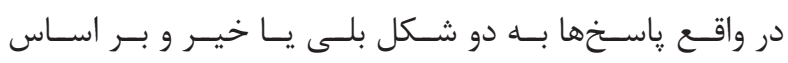

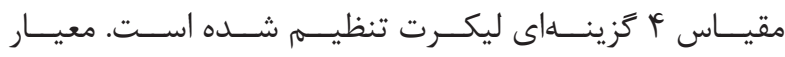

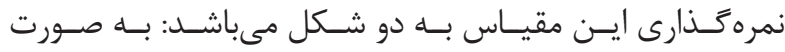

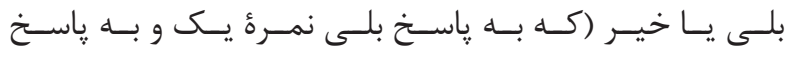

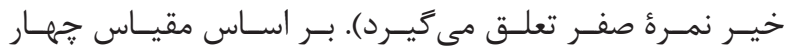

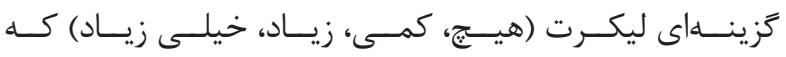

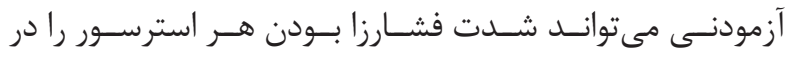

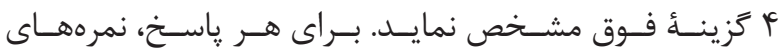

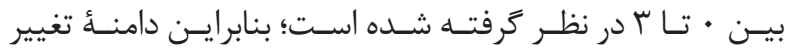

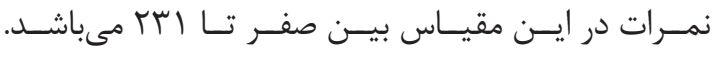

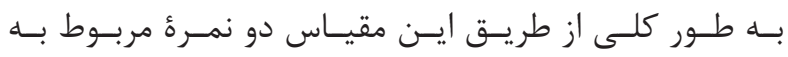

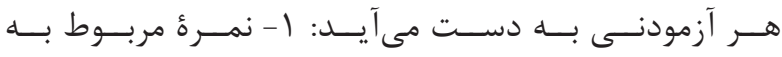

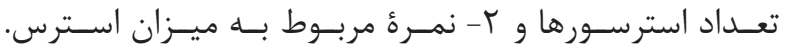

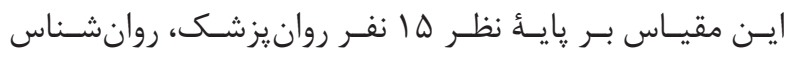

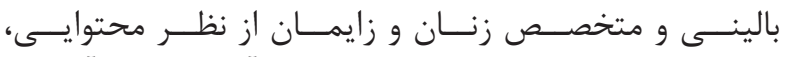

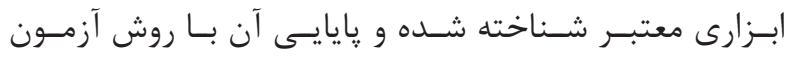

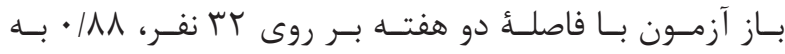

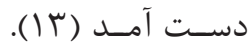


جدول ا- خلاصؤ تحليل واريانس يكطرفئ تعداد و شدت عوامل استرسزا در زوجهاى نابارور.

\begin{tabular}{|c|c|c|c|c|c|c|}
\hline سطع معنى & $\mathbf{f}$ & ميانكين & درجة آزانى & مجنموع مجورات & منبع تغييرات & متغير \\
\hline$\cdot / \cdot$ t人 & $r / r \cdot r$ & $\begin{array}{l}r \backslash \Lambda / r \backslash 0 \\
99 / r(Y)\end{array}$ & $\begin{array}{l}r \\
v \cdot \\
\text { vr }\end{array}$ & $\begin{array}{l}q \Delta f / g r q \\
q q \Delta r / \Lambda \Delta f \\
\vee q \cdot \Lambda / \Delta \cdot .\end{array}$ & دين كرورهها & \\
\hline $\begin{array}{l}\cdot 1 \cdot r \\
0^{*}\end{array}$ & $\Delta / \Gamma \mid$ & $\begin{array}{l}\text { IADI/AF. } \\
\text { rFA/GVT }\end{array}$ & $\begin{array}{l}r \\
v \cdot \\
\text { re }\end{array}$ & $\begin{array}{l}\Delta \Delta \Delta \Delta / \Delta T . \\
r F F \cdot V / I F r \\
r q 99 T / 99 r\end{array}$ & دين كرووهها & \\
\hline
\end{tabular}

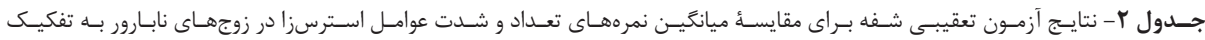

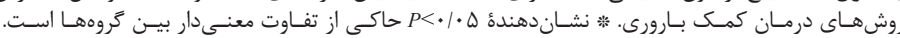

\begin{tabular}{|c|c|c|}
\hline واريانس مقابله & مياتكين مات & تعداد عوامل استرسزا \\
\hline $\begin{array}{l}1 / \cdots \\
. / 148 \\
. / 998\end{array}$ & 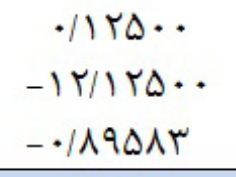 & $\begin{array}{c}\text { ICSI } \\
\text { IUI } \\
\text { بدون درمان }\end{array}$ \\
\hline $\begin{array}{l}1 / \cdots \\
\cdot 1 \cdot 91 \\
. / 991\end{array}$ & 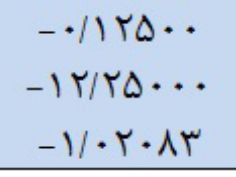 & $\begin{array}{c}\text { IVF } \\
\text { IUI } \\
\text { بدون درمان }\end{array}$ \\
\hline $\begin{array}{l}\cdot 1148 \\
\cdot 1 \cdot 91 \\
\cdot 1 \cdot 4 \mid\end{array}$ & 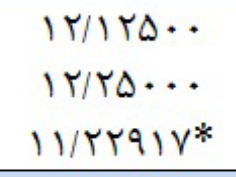 & $\begin{array}{c}\text { IVF } \\
\text { ICSI } \\
\text { بدون درمان }\end{array}$ \\
\hline $\begin{array}{l}\cdot / 99 \vee \\
. / 994 \\
. / \cdot 41\end{array}$ & 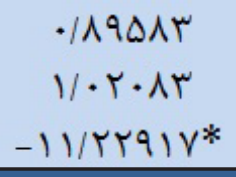 & $\begin{array}{ll}\text { IVF } & \\
\text { ICSI } & \\
\text { IUI } & \end{array}$ \\
\hline & & شدت عوامل استرسزا \\
\hline $\begin{array}{l}\cdot / 91 \cdot \\
\cdot / \cdot 14 \\
. / 9 V T\end{array}$ & $\begin{array}{l}r / \Lambda \cdots \\
-r f / \varepsilon r \Delta \cdots \\
r / f \mid \& \& V\end{array}$ & $\begin{array}{l}\text { ICSI } \\
\text { IUI } \\
\text { بدون درمان }\end{array}$ \\
\hline $\begin{array}{l}.191 \\
.1 \cdot r t \\
1 / \cdots\end{array}$ & 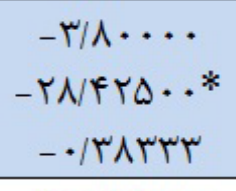 & $\begin{array}{c}\text { IVF } \\
\text { IUI } \\
\text { بدون درمان }\end{array}$ \\
\hline 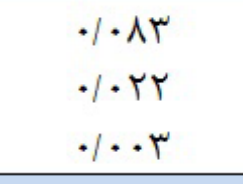 & $\begin{array}{l}r F / \varepsilon T \Delta \cdots \\
r N / F r \Delta \cdots * \\
r N / F I \& N^{*}\end{array}$ & $\begin{array}{c}\text { IVF } \\
\text { ICSI } \\
\text { بدون درمان }\end{array}$ \\
\hline $\begin{array}{l}. / 9 V r \\
1 / \cdots \\
.1 \cdots r\end{array}$ & $\begin{array}{c}-r / f I \& 8 V \\
• / r N r m \\
\text { - rN/fI\&V* }\end{array}$ & $\begin{array}{c}\text { IVF } \\
\text { ICSI } \\
\text { IUI }\end{array}$ \\
\hline
\end{tabular}




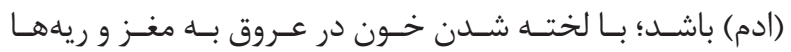

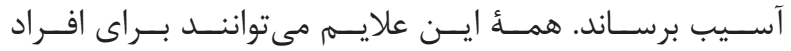

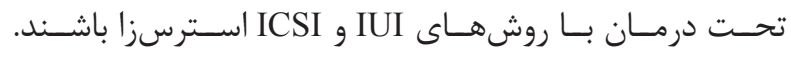

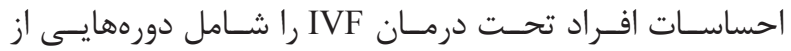

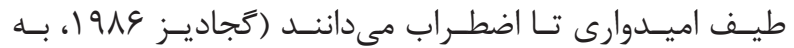

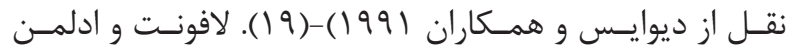

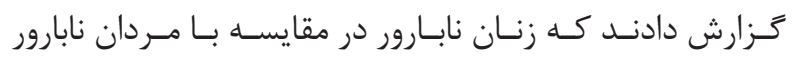

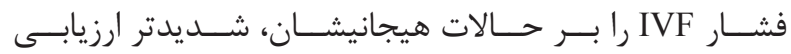

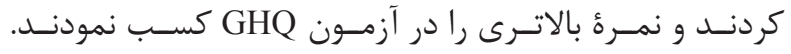

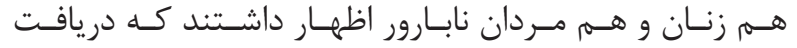

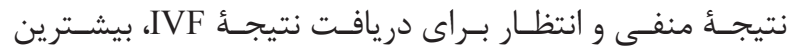

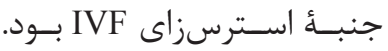

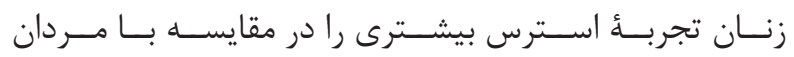

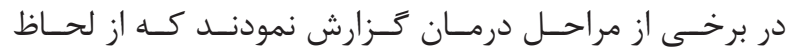

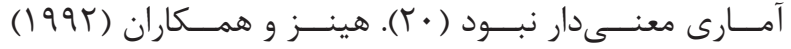

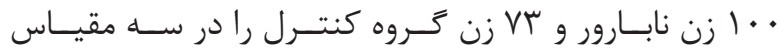

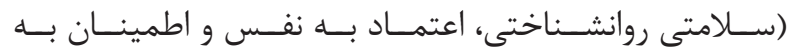

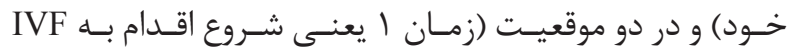

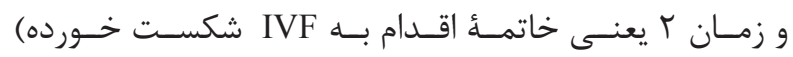

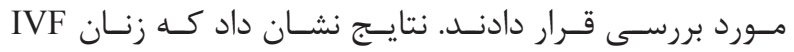

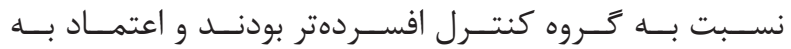

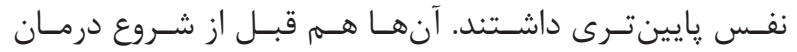

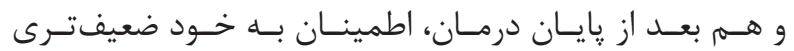

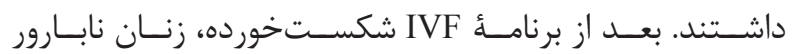

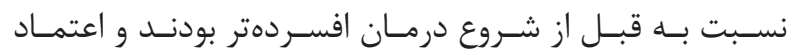

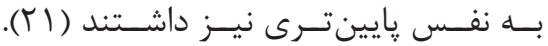

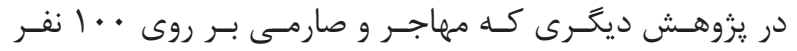

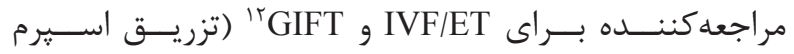

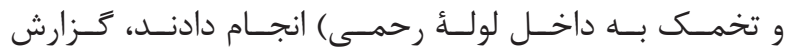

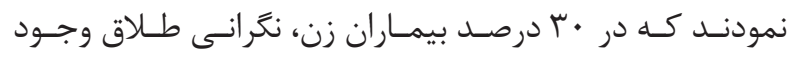

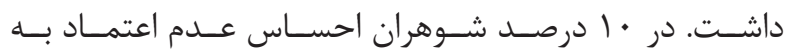

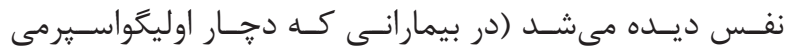

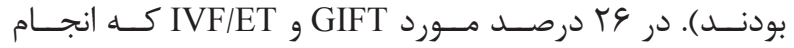

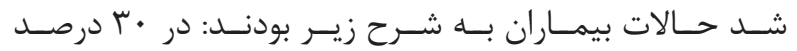

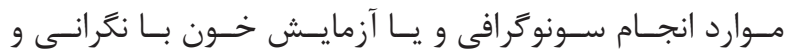

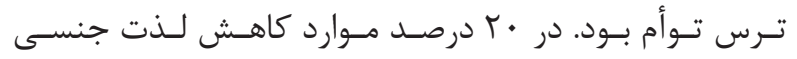

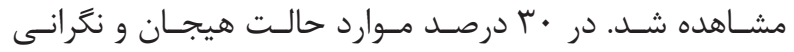

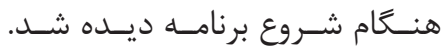

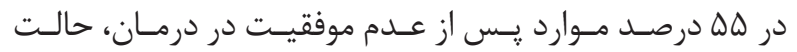

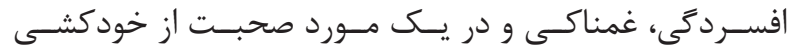

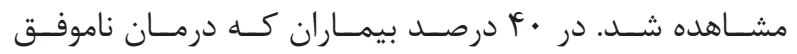

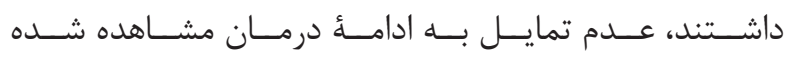

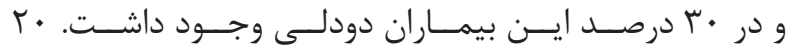

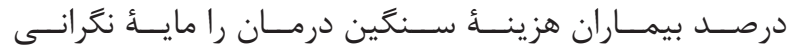

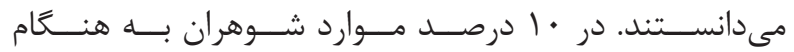

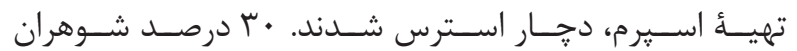

${ }^{11}$ Sexually transmitted diseases

${ }^{12}$ Gamete intrafallopian transfer

\section{بحث و نتيجه كيرى}

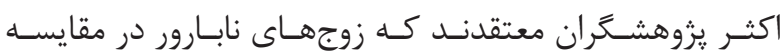

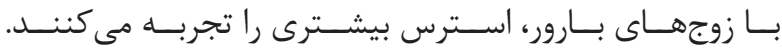

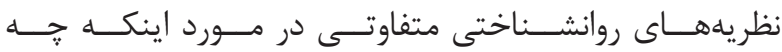

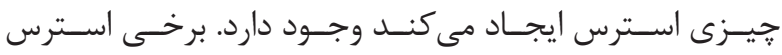

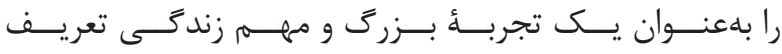

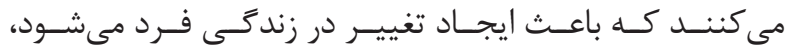

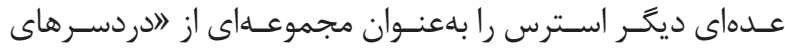

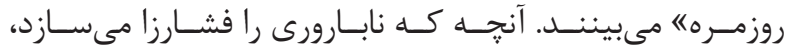

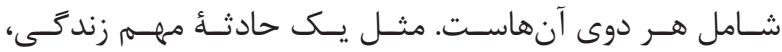

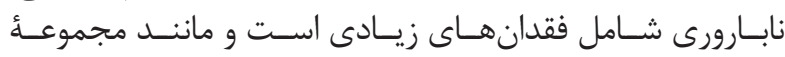

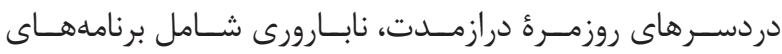

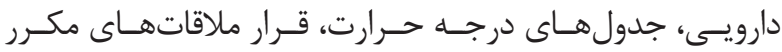

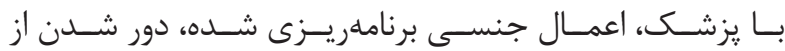

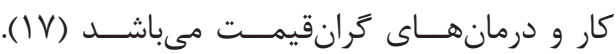

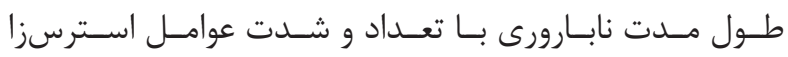

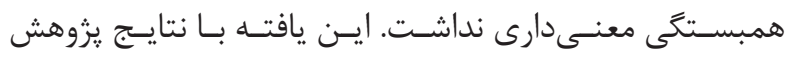

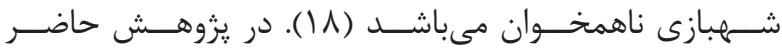

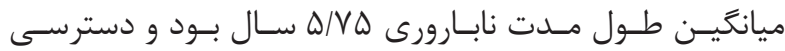

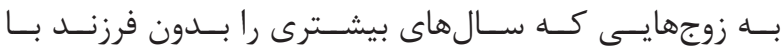

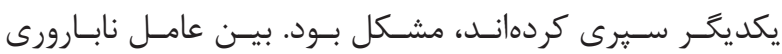

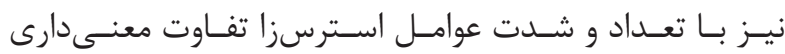

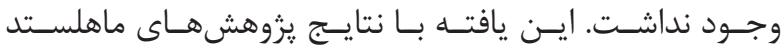

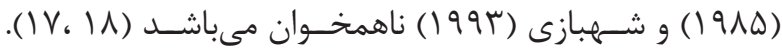

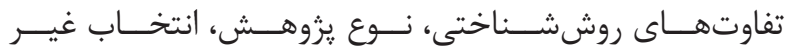

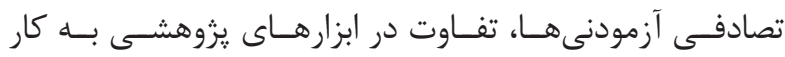

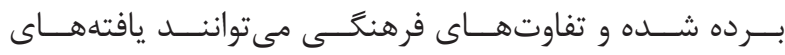

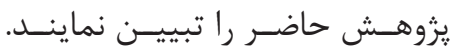

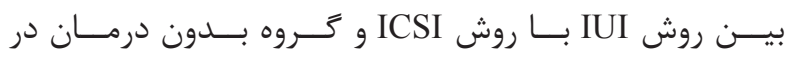

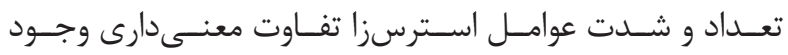

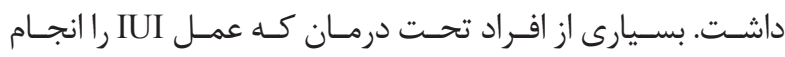

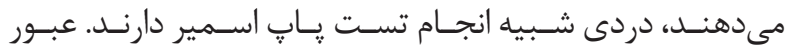

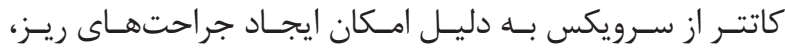

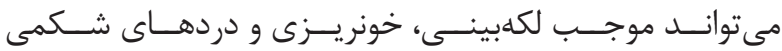

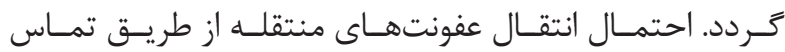

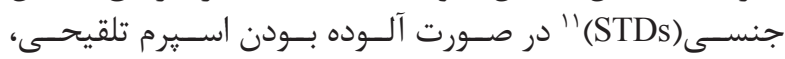

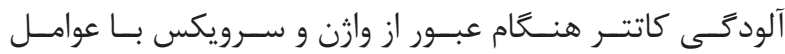

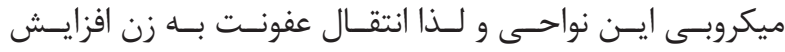

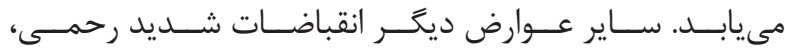

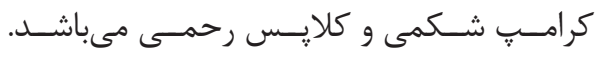

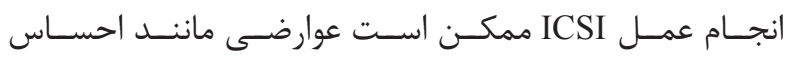

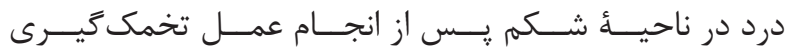

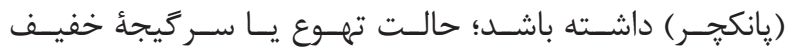

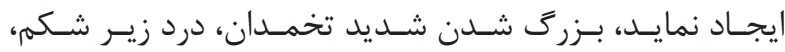

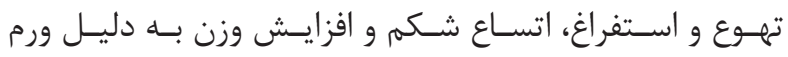


نشـان دهنــده سـطح آكاهـى باييـن تمامسى افـراد از مســائل

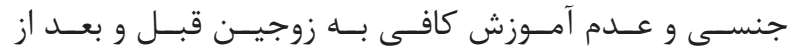

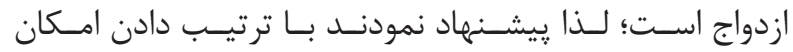

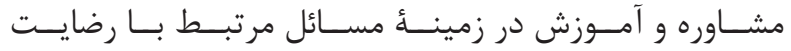

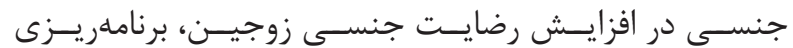

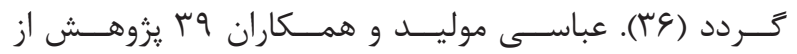

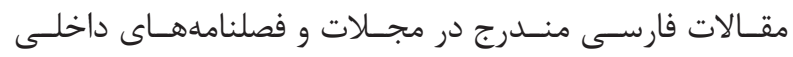

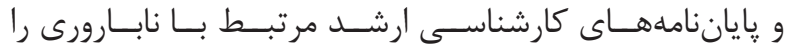

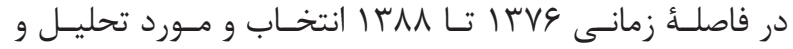

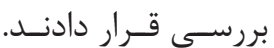

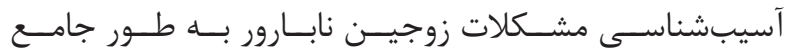

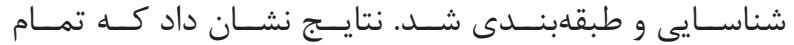

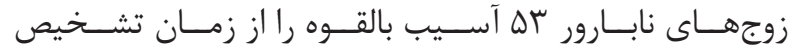

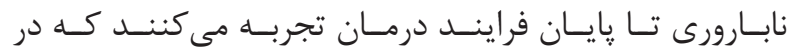

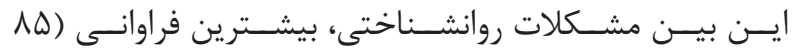

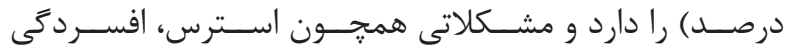

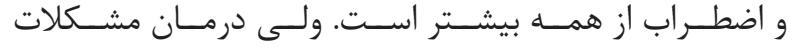

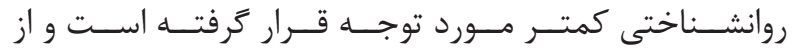

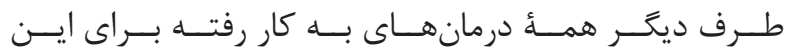

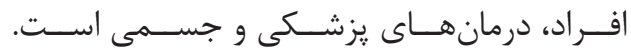

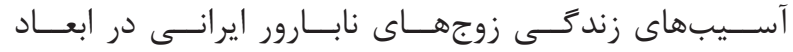

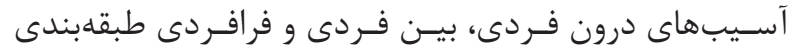

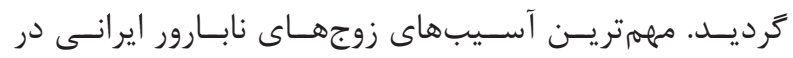

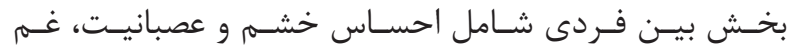

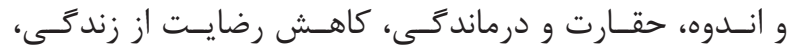

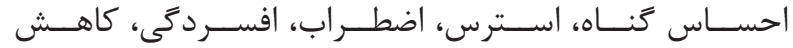

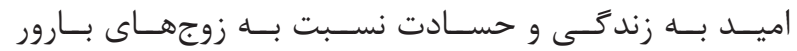

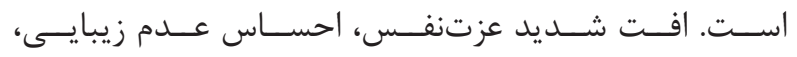

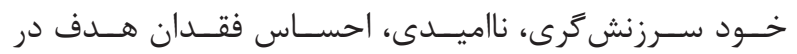

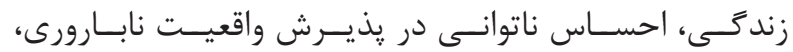

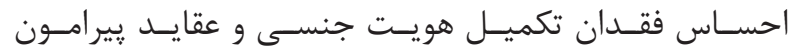

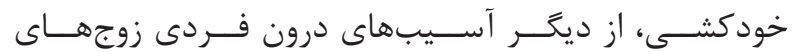

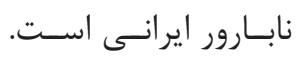

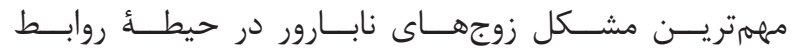

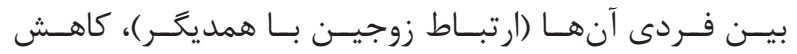

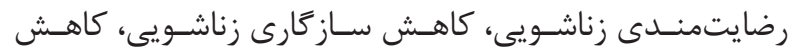

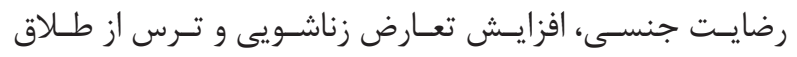

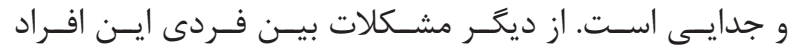

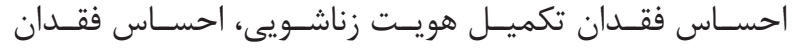

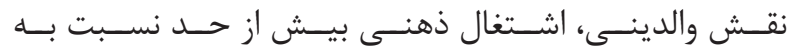

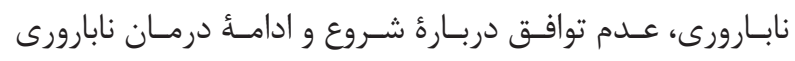

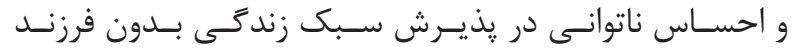

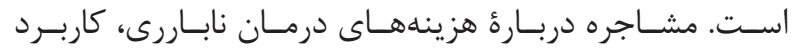

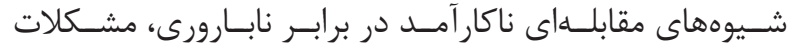

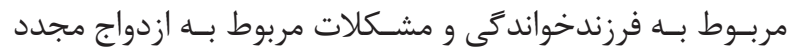

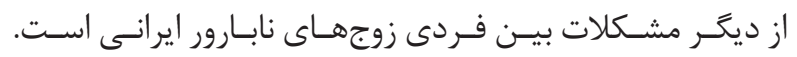

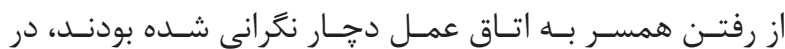

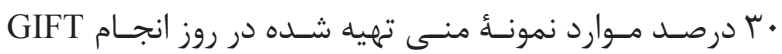

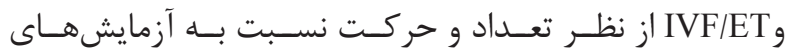

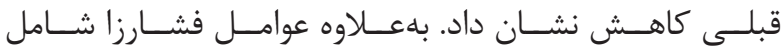

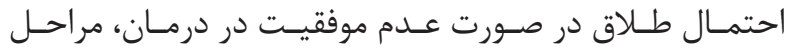

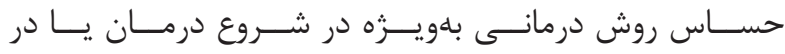

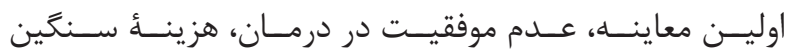

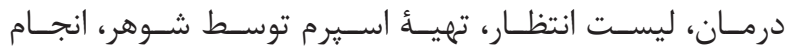

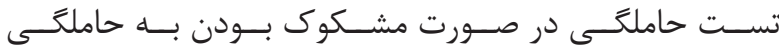

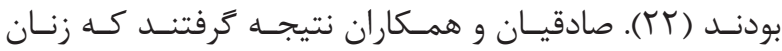

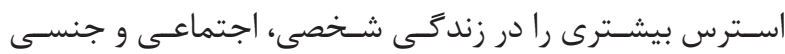

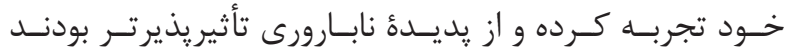

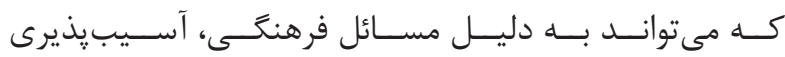

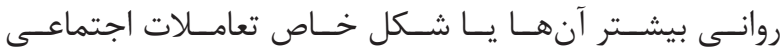

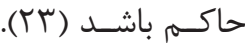

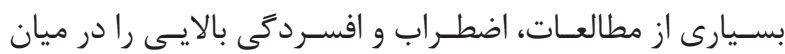

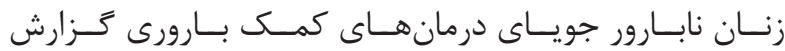

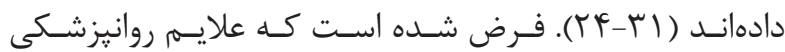

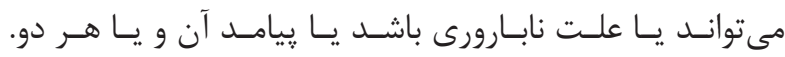

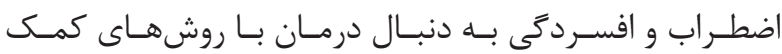

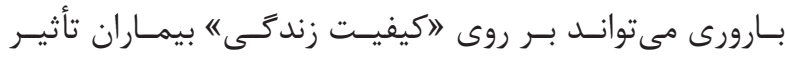

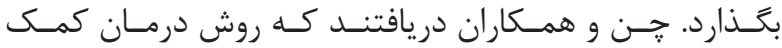

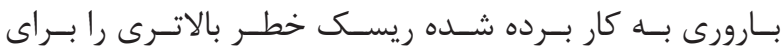

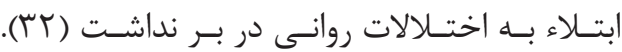

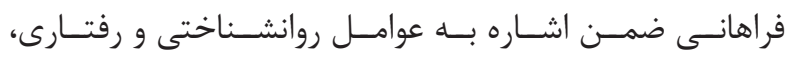

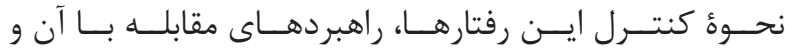

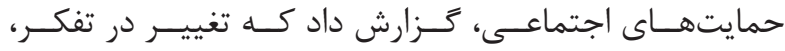

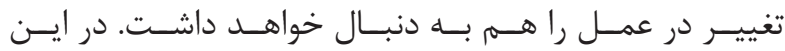

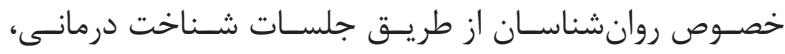

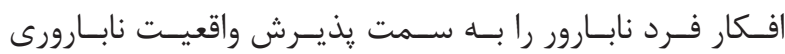

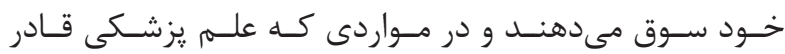

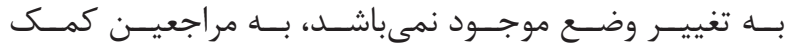

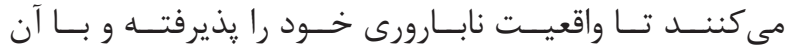

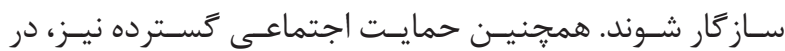

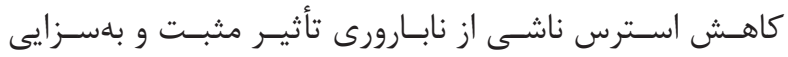

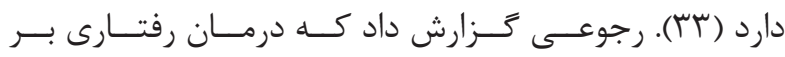

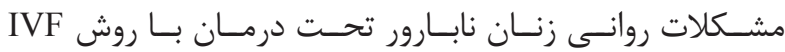

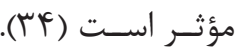
طاهـــى ينـاه و همــكاران (بــهـ نقـلـل از خداكرمسى و همـكاران)

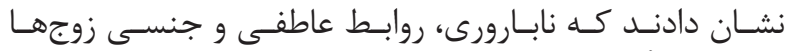

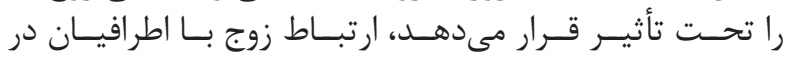

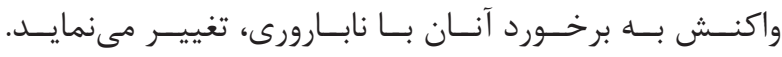

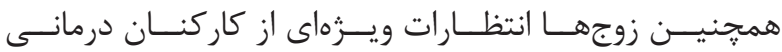

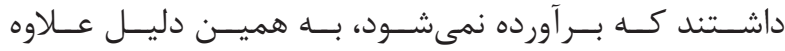

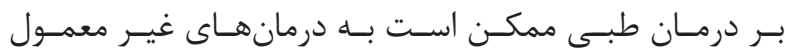

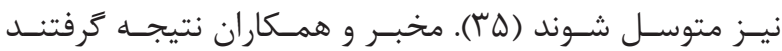

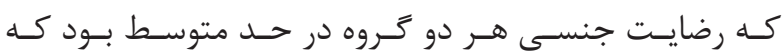


نابـارور غيــر مراجعــه كنـــده محسـدود مى كنــد. مقطعـى بــودن

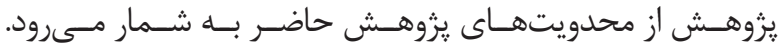

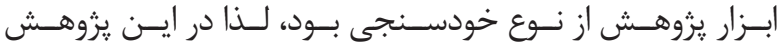

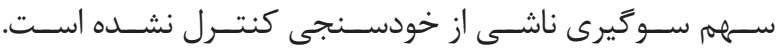

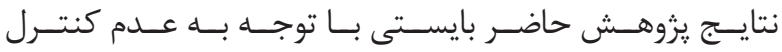

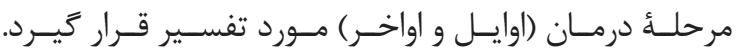

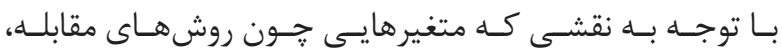

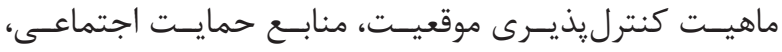

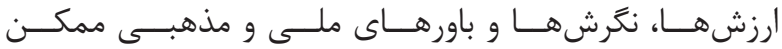

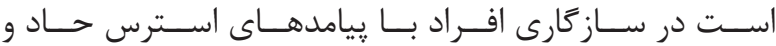

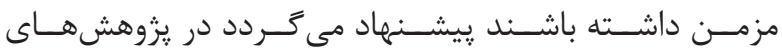

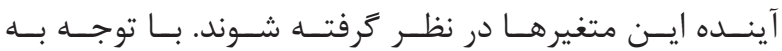

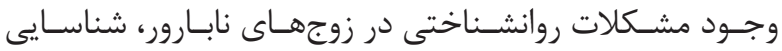

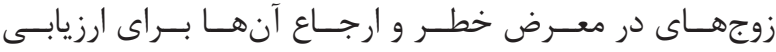

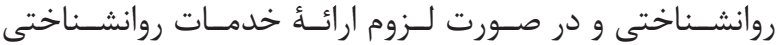

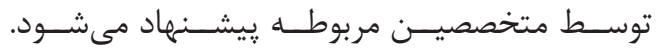

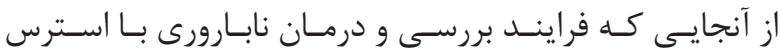

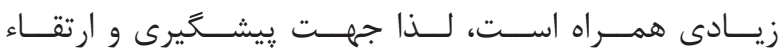

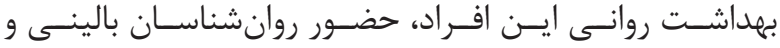

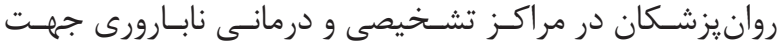

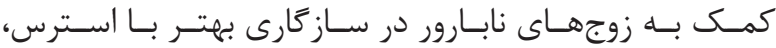

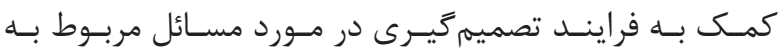

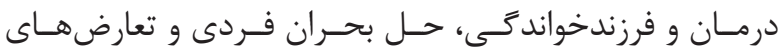

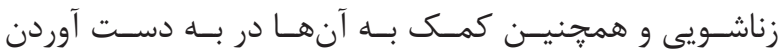

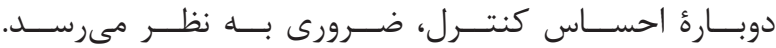

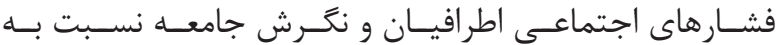

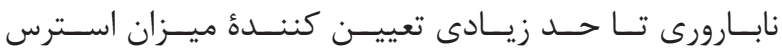

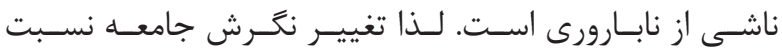

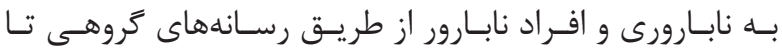

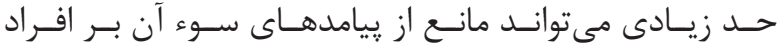

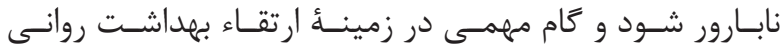

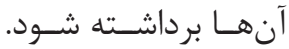

1. Dadfar M, Kazemi H, Kazem Zadeh Atoofi M, Kolivand PH. Psychological aspects of infertility. Tehran. Mirma. 2014.

2. Spiroff L, Fritz MA. Clinical gynecology endocrinology and infertility. $17^{\text {th }}$ ed. New York: Lippincott Williams and Wilkins. 2005; p. 12.

3. Berek JS, Novak E. Berek and Novak's Gynecology. 14th ed. New York: Philadelphia: Lippincott Williams and Wilkins. 2006; p. 506.

4. Peterson BD. Newton CR, Rosen KH. Examining
آسـيبهاى فرافـردى و اجتماعى و محيـط يِيرامـون زوجهـاى

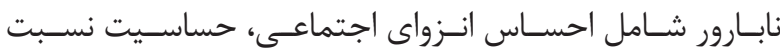

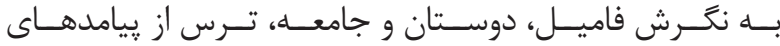

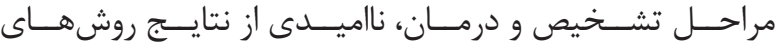

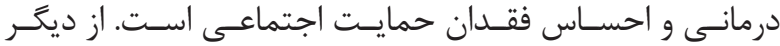

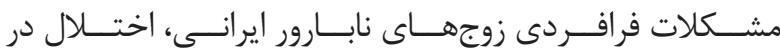

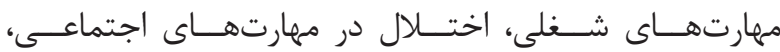

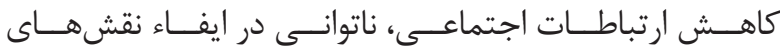

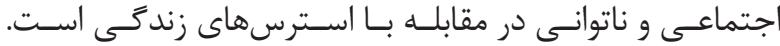

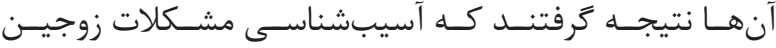

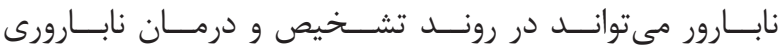

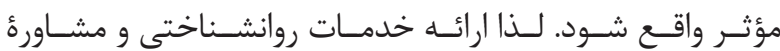

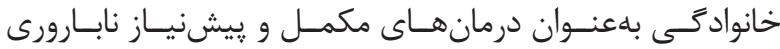

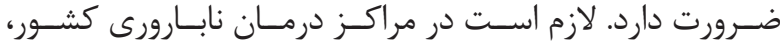

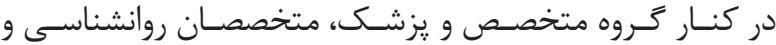

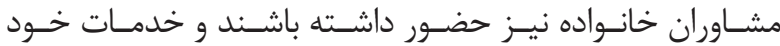

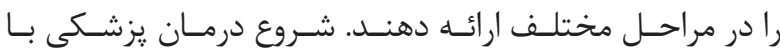

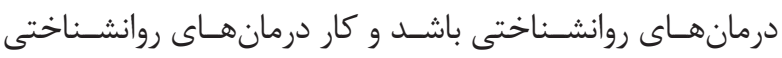

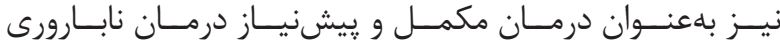

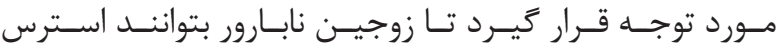

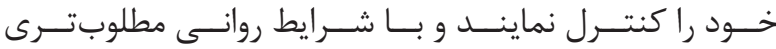

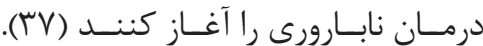

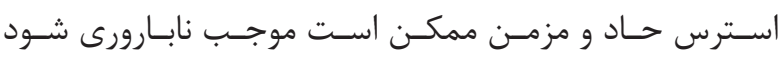

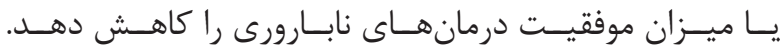

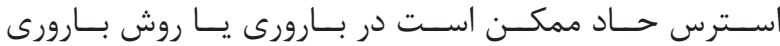

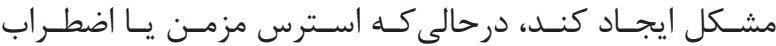

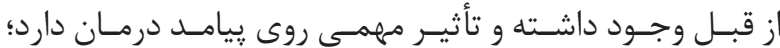

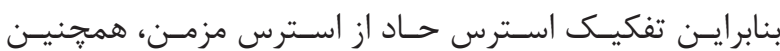

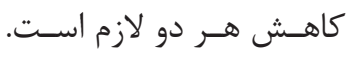

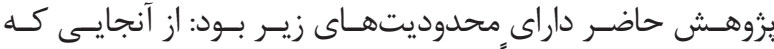

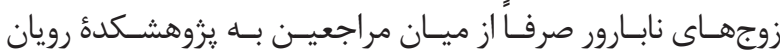

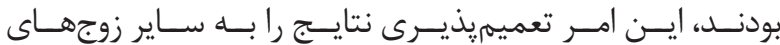

منابع

congruence between partners perceived infertilityrelated stress and its relationship to marital adjustment and depression in infertile couples. Fam Process. 2003; 42(1): $59-70$.

5. Peterson BD, Newton CR, Rosen KH, Skaggs GE. Gender differences in how men and women who are referred for IVF cope with infertility stress. Human Reproduction. 2006; 21(9): 2443-9.

6. Cleveland Clinic. Infertility: Is it stress related? http:// my.clevelandclinic.org/health/diseases conditions/hic Infertility Is it Stress Related. 
7. Dadfar M, Eftekhar Ardebili M. Psychological dimensions of infertility. Abstract book of psychological aspects of infertility symposium. sid ave sina research center. 2001.

8. Dadfar M, Dadfar F. Comparative study of stress in infertile and fertile women. Abstract book of the first congress of role of woman in family and community health. alzahra university. 2009.

9. Dadfar F, Dadfar M. Comparative study of stress in infertile and fertile men. Abstract book of the forth congress of family and sexual health. shahed university. 2009; p. 20-2.

10. Dadfar M, Dadfar F, Comparative study of mental health in infertile and fertile couple. Abstract book of the First Congress of maintenance of fertility and the 15th Annual Congress news about fertility and infertility of SID Ave Sina Research Center. Shahid Beheshti Medical Sciences University. 2009; p. 23-4.

11. Pazandeh F, Sharghi Someeh N, Karami Noori R, Alavi Majd J. Relationship infertility with well-being and satisfaction of marriage. Pajohanden. 2004; 9(6): 9-15.

12. Campagne DM. Should fertilization treatment start with reducing stress? Hum Reprod. 2006; 21(7): 1651-8.

13. Pahlavani H, Malakouti K, Shahrokh Tehrani Nejad E. Stressors, their coping strategies, and relation to mental health in infertile individuals. IJPCP. 2002; 7(4): 79-87.

14. Abbey A, Halman LJ, Andrews FM. Psychological treatment and demographic predictors of the stress associated with infertility. Fertil Steril. 1992; 57(1): 122-7.

15. Demyttenaere K, Nijs P, Kiebooms GE, Konincky PR. The effect of a specific emotional stressor and prolactin, cortisol, and testosterone concentration in women varies with their trait anxiety. Fertil Steril. 1989; 52(6): $942-8$.

16. Wright J, Duchesne C, Sabourin S, Bissonnette F, Benoit J, Giraod Y. Psychological distress and infertility men and women respond differently. Fertil Steril. 1991; 55: $100-8$.

17. Mahlsted PP. Psychological component of infertility. Fertil Steril. 1985; 43: 335-49.

18. Shahbazi L. Study and comparison of depression in women with infertility problem and women' partners with infertility problem. Master Thesis. Tehran. Iran University of Medical Sciences, School of Nursing and Midwifery. 1993.
19. Davis D, Derman C. Coping strategies of infertile women. J Obstet Gynecol Neonatal Nurs. 1991; 20: 221-8.

20. Laffont I, Edelmann RJ. Psychological aspect of invitro fertilitization: a gender comparison. J Psychosom Obstet Gynaecol. 1994; 15(2): 85-92.

21. Hynes GJ, Callan VG, Terry DJ, Gallois C. The psychological well being of infertile women after a failed IVF attempt. Br J Med Psychol. 1992; 65: 269-78.

22. Mohajeri H, Saremi A. Stress of IVF. J Drug Therap. 1991; 8(96): 14-6.

23. Sadeghian A, Heydarian Pour A, Abed F. Comparison psychological problem of male and female that Referred to infertility clinic of Fatemieh Hospital in Hamedan in 2004. J Arak Univ Med Sci. 2006; 14(9): 1-6

24. Domar AD, Broome A, Zuttermeister PC, Seible M, Friedman R. The prevalence and predictability of depression in fertile women. Fertil Steril. 1992; 58: 1158-63.

25. Demyttenaere K, Bonte L, Gheldof M, Vervaeke M, Meuleman C, Vanderschnerem D, et al. Coping style and depression level influence outcome in invitro fertilization. Fertil Steril. 1998; 69: 1026-33.

26. Lukse MP, Vacc NA. Grief, depression and coping in women undergoing infertility treatment. Obstet Gynecol. 1999; 93(2): 245-51.

27. Oddens BJ, den Tonkelaar I, Nieuwenhuyse H. Psychosocial experiences in women facing fertility problems: a comparative survey. Hum Reprod. 1999; 14(1): 255-61.

28. Hjelmstedt A, Andersson L, Skoog-Svanberg A, Bergh T, Boivin J, Collins A. Gender differences in psychological reactions to infertility among couples seeking IVF and ICSI treatment. Acta Obstet Gynecol Scand. 1999; 78(1): 42-8.

29. Matsubayashi H, Hosaka T, Jzumi S, Suzuki T, Makino T. Emotional distress of infertile women in Japan. Hum Reprod. 2001; 16(5): 966-9.

30. Anderson KM, Sharpe M, Rattray A, Irvine DS. Distress and concerns in couple referred to a specialist infertility clinic. J Psychosom Res. 2003; 54(4): 353-5.

31. Monga M, Alexanderescu B, Katz SE, Stein M, Ganiats T. Impact of infertility on quality of life, marital adjustment and sexual function. Urology. 2004; 63(1): 126-30. 
32. Chen TH, Chang Sh P, Tsai Ch F, Juang KD. Prevalence of depressive and anxiety disorders in an assisted reproductive technique clinic. Hum Reprod. 2004; 19(10): 2313-8.

33. Farahani MN. Psychological aspects of coping and adjustment with infertility and role of psychologist. Journal of Reproduction and Infertility. 2001;2(4): 52-9.

34. Rojouie M. Effect of behavioral therapy on the mental health of infertile women treated with IVF. Master thesis. Tehran. University of Social Welfare and Rehabilitation Sciences. 1997.
35. Khodakarami N, Hashemi S, Sedigh S, Hamdieh M, Tehri Panah R. Life experience with infertility: a phenomenological study. J Reprod Infertil. 2009; 10(4): 287-98.

36. Mokhber Gh, Shakeri MT, Joniedi E, Noorie Saadoldin Sh. Sexual satisfaction in fertile and infertile women attending state clinics in Mashhad. J Reprod Infertil. 2009; 10(4): 269-78.

37. Abbasi MH, Ghomarani A, Fatehi Zadeh M. The pathology of Iranian infertile couples life. Teb va Tazkieh. 2009; 72: 8-20. 